\title{
Biological Therapeutic Targets in Muscle-Invasive Bladder Cancer
}

Amal Abd El hafez ${ }^{1,2^{*}}$

${ }^{1}$ Pathology Department, Faculty of Medicine, Mansoura University, Egypt

${ }^{2}$ Basic Medical Sciences Department, College of Medicine Dar Al Uloom University, KSA, Saudi Arabia

"Corresponding author: Amal Abd El hafez, Pathology Department, Faculty of Medicine, Mansoura University, Egypt and Basic Medical Sciences Department, College of Medicine Dar Al Uloom University, KSA, Saudi Arabia, Tel: 966557662665; E-mail: amalabdelhafez@gmail.com

Rec date: Oct 31, 2014, Acc date: Nov 02, 2014, Pub date: Nov 04, 2014

Copyright: (C) 2014 Abd El hafez A. This is an open-access article distributed under the terms of the Creative Commons Attribution License, which permits unrestricted use, distribution, and reproduction in any medium, provided the original author and source are credited.

\section{Editorial}

With a worldwide estimate of about 300,000 new cases diagnosed every year, bladder cancer represents the second most common genitourinary malignancy after prostatic carcinoma and the second most common cause of genitourinary cancer-related deaths [1]. There are two major clinico-pathological subtypes of bladder cancer: nonmuscle-invasive cancer (NMIBC), which frequently recurs but often has good prognosis; and muscle-invasive bladder cancer (MIBC) with poor prognosis [2]. At initial diagnosis, $75 \%$ of patients present with NMIBC and $25 \%$ of patients have MIBC or metastatic disease [3]. Approximately $10-20 \%$ of NMIBC progresses to MIBC and $10-30 \%$ recurs $[3,4]$. Of the patients initially presenting with MIBC, about $50 \%$ relapse with metastatic disease [4]. When there is local or distant metastasis, MIBC is mostly incurable (90\%) and only $10 \%$ of patients respond to systemic chemotherapy following radical cystectomy [2]. Although intra-vesical immuno- and chemotherapy, surgery, and systemic chemotherapy are all critical elements in the management of patients with bladder cancer, novel approaches for the management of patients with MIBC are still desperately needed to improve patient outcome.

Histologically, bladder cancer originates from the transitional epithelium that is composed of 3 to 6 layers of basal, intermediate, and multinucleate umbrella cells. So, there are three types of bladder cancer namely: transitional cell carcinomas, which account for more than $90 \%$ of bladder tumors; squamous cell carcinomas, which constitute about $8 \%$ of bladder tumors and are usually associated with long-term infection or irritation of the urothelium in addition to adenocarcinomas, which account for about $2 \%$ of bladder neoplasms and arise from the glandular (secretory) cells and often have urachal or metaplastic origin [5].

Tumors are considered as phenotypic manifestations of genomic, genetic, and epigenetic abnormalities. At the heart of this concept is the critical involvement of intrinsic biological properties of an affected tissue to oncogenic processes [6]. Urothelium is one of the slowest cycling epithelia in the body, with a turnover rate of about 200 days and a tritium-thymidine labeling index of less than $0.01 \%$, a matter that personifies a unique biological milieu for cellular transformation [7], entailing several molecular alterations and complex biological pathways that regulate cellular processes, such as proliferation, differentiation, angiogenesis, metastasis and apoptosis. Therefore, discovering new biological targets, may improve the clinical prediction and the personalization of therapeutic approaches of bladder cancer patients with the purpose of reducing the risk of progression.

Recent advances in the understanding of the pathogenesis of bladder cancer at the molecular level have provided a significant number of specific biological molecular targets for therapy and have shown the importance of individualized therapy according to the molecular profile exhibited by the tumor cells. Of several biological markers that can be targeted by small molecules or monoclonal antibodies are the human epidermal growth factor receptor 2 (Her-2/Neu also known as erythroblastic leukemia viral oncogene homolog 2; erbB2), vascular endothelial growth factor (VEGF), fibroblast growth factor receptor-3 (FGFR3), mutant p53/pRb and PTEN (phosphatase and Tensin Homolog), survivin, and nuclear factor- $\kappa B(N F \kappa B)[8,9]$.

The most intensively studied molecular biomarker for bladder cancer is p53. It plays a vital role in the regulation of cell cycle and is important for genetic stability, cell proliferation, apoptosis, and inhibition of angiogenesis. A defect in p53 leads to loss of p53dependent apoptosis and gives a proliferation advantage [3]. Inactivation of both $\mathrm{p} 53$ and $\mathrm{pRb}$, a prevailing paradigm previously proposed for MIBC tumorigenesis, is found to be necessary but insufficient to initiate bladder cancer. Instead, downregulation in $\mathrm{p} 53 / \mathrm{pRb}$ co-deficient urothelial cells of $\mathrm{p} 107$, a pRb family member, is associated with the genesis of the MIBC [7]. Some studies found that altered p53 gradually increases from normal urothelium to NMIBC to MIBC, and significantly associates with tumor stage, grade, lymphovascular invasion, nodal metastases and disease recurrence [3]. About $50 \%$ of the MIBC harbor p53 mutations and aberrant pRb expression simultaneously and these two alterations are more significantly associated with poor prognosis and patient survival than one alteration alone. Additionally, p53 deficiency seems to be capable of cooperating with that PTEN, located on human chromosome 10 (a lipid phosphatase that dephosphorylates phosphoinositide-3,4,5triphosphate; PI3P), in eliciting invasive bladder cancer [7]. In vitro inactivation of PTEN, was found to enhance oncogenic transformation and reintroduction of PTEN in cells lacking it, was suppressing to cell growth. Similarly, the findings that deficiency of p53 cooperates with that of $\mathrm{pRb}$ and $\mathrm{p} 107$ or PTEN to induce invasive urothelial carcinomas suggested that replacing and/or restoring the function of one or more of these tumor suppressors could adjunct to radical cystectomy and chemotherapy. Viral vector-based gene therapies based on $\mathrm{pRb}$ or $\mathrm{p} 53$ alone have already been tested for human bladder cancer, showing early promise [7].

Her-2/Neu, encoded by c-erbB2 gene localized to chromosome $17 \mathrm{q}$, is a $185 \mathrm{kDa}$ membrane-spanning glycoprotein with tyrosine kinase activity. Unlike other EGFRs, Her-2/neu does not have its own ligand; instead, it heterodimerizes with other activated EGFR members to enhance and prolong their mitogenic signaling. Thus, Her-2/Neu plays a basic role in cell growth, differentiation survival, and migration, and abnormal activation of Her-2/Neu leads to oncogenic transformation of the cell. Her-2/Neu appears to play role in the pathogenesis of bladder cancer and its protein overexpression has been considered as a 
bad prognostic factor in MIBC. Lately, anti- Her-2/Neu antibody, also known as transtuzumab or Herceptin, has shown its efficiency in these tumors therapy. Consequently, assessment of Her-2/Neu status has assumed therapeutic significance. Her-2/Neu protein overexpression is believed to arise from a combination of two mechanisms: gene amplification which is not a common mechanism in bladder cancer $[10,11]$; and upregulated transcription, which reflects the nature of the Her-2/Neu protein as a growth factor. In a recent study, Her-2/Neu was observed in $62.5 \%$ of MIBC. Moreover, a significant direct association was observed between Her-2/Neu protein overexpression and both increasing grade of carcinoma, depth of tumor invasion and with the reduced differentiation grade reflecting the aggressiveness of the tumor cells. Therefore, Her-2/Neu overexpression was represented as a prognostic factor for adverse disease outcome and offered supplementary information for prognosis in patients with MIBC [12]. Yet, in cancer cells overexpressing $\mathrm{Her}-2 / \mathrm{Neu}$ due to gene amplification, high basal autophosphorylation of Her-2/Neu promotes oncogenic activities including metastasis and therapeutic resistance in MIBC, thus, the adjunctive use of erbB2 inhibitors may improve chemo/radiotherapy sensitivity [8].

An important determinant of angiogenesis is the vascular endothelial growth factor (VEGF) family that promotes endothelial mitogenesis and migration, extracellular matrix remodeling, increased vascular permeability, and maintenance of newly formed vasculature. Recently, VEGF was shown to be overexpressed in a large number of patients treated with radical cystectomy for urothelial carcinoma of the bladder (86\%). Higher VEGF expression was associated with increasing tumor stage, grade, progression, nodal metastasis and recurrence in bladder cancer but also with altered expression of $\mathrm{p} 21$, p27, RB, cyclin E1, and Ki-67, suggesting complex interactions between different pathways. These findings support the role of VEGF in bladder tumorigenesis and further support it as a potential target for therapy. Moreover, increased VEGF levels can result in increased vascular permeability and interstitial fluid pressure, impairing chemotherapy delivery. Adding anti-VEGF to chemotherapeutic regimens might lead to improved responses [3]. One study demonstrated that VEGF-A, a member of the PDGF/VEGF family, which promotes angiogenesis through nitric oxide synthase, was significantly up-regulated in bladder in cancer tissue compared to nontumor tissue suggesting that VEGF-A might be a commonly overexpressed gene in MIBC [4]. Moreover, the pattern of VEGF expression in MIBC correlated with the expression of the transcription factor hypoxia inducible factor-1 alpha (HIF-1 alpha) and with hypoxia-inducible factor-2 alpha (HIF-2 alpha). Therefore, HIF-1 alpha and HIF-2 alpha, are important cofactors in the regulation of VEGF in bladder cancer and are therapeutic targets in this disease [13].

$\mathrm{NF \kappa B}$ is a transcription factor involved in cellular response to various extracellular stimuli such as stress and cytokines. In cancer cells, NFKB is often activated aberrantly, promoting the invasion, metastasis, and survival of these cells. Indeed, NFkB overexpression is associated with poor prognosis and with chemo/radiotherapy resistance in various malignancies including MIBC especially, in cooperation with Her-2/Neu overexpression. These data strengthen the case for a putative role of NFkB inhibitors, of which many types are under clinical investigation, in overcoming chemo/radiotherapy resistance in MIBC overexpressing NFkB. Among the small molecules currently being investigated in clinical studies, heat shock protein 90 (Hsp90) inhibitors that can simultaneously block the oncogenic activity of both of both NFKB and Her-2/Neu [8].
Survivin, a member of the inhibitor of apoptosis protein family, is reportedly associated with local therapeutic failure in bladder cancer patients treated with chemo/radiotherapy. Recently, a small molecule inhibiting survivin has been identified and was expected to sensitize MIBC to chemo/radiotherapy in clinical settings [8].

The identification of mutations of FGF receptor 3 (FGFR3) in most noninvasive bladder tumors and the recent finding of overexpression of this receptor not only in superficial tumors but also in many MIBC has generated optimism that therapies targeting this receptor tyrosine kinase may have major application in the treatment of urothelial cancers. Recent preclinical evaluations of FGFR3 as a therapeutic target have provided a strong force for the development of targeted agents for clinical use [14].

Since, MIBC is biologically and clinically distinct, it seems worthwhile to understand its biology and genetics in order to identify and define the role of molecules and pathways appropriate for noveltargeted therapies.

\section{References}

1. Voutsinas GE, Stravopodis DJ (2009) Molecular targeting and gene delivery in bladder cancer therapy. J BUON 14 Suppl 1: S69-78.

2. Chan KS, Espinosa I, Chao M, Wong D, Ailles L et al. (2009) Identification, molecular characterization, clinical prognosis, and therapeutic targeting of human bladder tumor-initiating cells. Proc Natl Acad Sci USA 106: 14016-14021.

3. Youssef RF, Lotan Y (2011) Predictors of outcome of non-muscle-invasive and muscle-invasive bladder cancer. ScientificWorldJournal 11:369-381.

4. Zhang S, Liu Y, Liu Z, Zhang C, Cao 1, et al. (2014) Transcriptome profiling of a multiple recurrent muscle-invasive urothelial carcinoma of the bladder by deep sequencing. PLoS One 9: e91466.

5. Vishnu P, Mathew J, Tan WW (2011) Current therapeutic strategies for invasive and metastatic bladder cancer. Onco Targets Ther 4: 97-113.

6. Stratton MR, Campbell PJ, Futreal PA (2009) The cancer genome. Nature 458: 719-724.

7. Wu XR (2009) Biology of urothelial tumorigenesis: insights from genetically engineered mice. Cancer Metastasis Rev 28: 281-290.

8. Koga F, Yoshida S, Tatokoro M, Kawakami S, Fujii Y, et al. (2011) ErbB2 and $\mathrm{NF} \hat{I}^{\circ} \mathrm{B}$ overexpression as predictors of chemoradiation resistance and putative targets to overcome resistance in muscle-invasive bladder cancer. PLoS One 6: e27616.

9. Ghosh M, Brancato SJ, Agarwal PK, Apolo AB (2014) Targeted therapies in urothelial carcinoma. Curr Opin Oncol 26: 305-320.

10. Jimenez RE, Hussain M, Bianco FJ, Jr. Vaishampayan U, Tabazcka P et al. (2001) Her-2/neu overexpression in muscle-invasive urothelial carcinoma of the bladder: prognostic significance and comparative analysis in primary and metastatic tumors. Clin Cancer Res 7: 2440-2447.

11. Krüger S, Weitsch G, Büttner H, Matthiensen A, Böhmer T, et al. (2002) HER2 overexpression in muscle-invasive urothelial carcinoma of the bladder: prognostic implications. Int J Cancer 102: 514-518.

12. Shawky AA, Abd El hafez A, Elosaily G, Al-Matubsi HY, Farahat A (2013) Her-2/Neu Overexpression in Invasive Urinary Bladder Carcinoma among a Cohort of Egyptian Patients. World J Nephrol Urol 2: 70-75.

13. Jones A, Fujiyama C, Blanche C, Moore JW, Fuggle $S$ et al. (2001) Relation of vascular endothelial growth factor production to expression and regulation of hypoxia-inducible factor-1 alpha and hypoxia-inducible factor-2 alpha in human bladder tumors and cell lines. Clin Cancer Res 7: 1263-1272.

14. Knowles MA (2008) Novel therapeutic targets in bladder cancer: mutation and expression of FGF receptors. Future Oncol 4: 71-83. 